Acta Physica Academiae Scientiarum Hungaricae, Tomus 24 (4), pp. 373-380 (1968)

\title{
AN APPROXIMATION OF THE MANY-ELECTRON PROBLEM BY THE UNIFICATION OF THE CHARGE CLOUD
}

\author{
By \\ G. Náray-Szabó \\ DEPARTMENT OF GENERAL AND INORGANIC CHEMISTRY, ROLAND EÖTVÖS UNIVERSITY, BUDAPEST
}

(Presented by B. Lengyel. - Received 1. VI. 1967)

Similar to the formation of first-order density matrices we can construct three-dimensional functions (here called density functions) from the $3 N$-dimensional solution of the manybody problem. If we choose the Hamiltonian in a suitable form the energies of atoms can be approximately calculated with the aid of these functions. Depending on the form of the wave function the deviation from the Hartree-Fock method is some tenths or some per cent. The approximation is the most accurate and the most simple in the spherically symmetrical case. The discussion is possible only for stationary problems.

\section{Introduction}

It is well known that one of the main difficulties in the many-body problem is due to the large number of variables. The approximations used today are so complicated that trying to get an exact relation between a certain physical constant of the system and the atomic parameters (atomic number, principal quantum number, number of electrons, etc.) is almost impossible. Though, by the aid of high-speed computers very great acceuracy is reached, the development of an approximate method which is less precise but still generally adaptable for more complicated atoms, also, is not without interest.

In the literature we can find a great number of attempts which are confined to the global treatment of the system as a whole, in place of the precise description of each of the particles. This means, from a mathematical point of view, that they work with certain averaged functions by the aid of which the energy can be calculated with sufficient accuracy. The oldest of these is the Thomas-Fermi model. The electrons of the system which can be described by Fermi-Dirac statistics are treated as a degenerated electron gas at the absolute zero temperature in which the distribution of the particles is continuous. This assumption is reasonable in the case of a great number of particles only, so the error made in the case of the light atoms is considerably great. A comprehensive description of the method is given in the book by Gombás [l].

In the statistical theory of fluids we can simplify the problem by the description of a given canonical $N$-particle ensemble with the aid of averaged functions [2]. Similarly, the use of the reduced density matrices is widely 
accepted in quantum mechanics. In this case not the wave function but the density operator is averaged [3-4]. Attempts have been made at the $a$ priori calculation of the first and second-order density matrices but these lead to very complicated expressions [5-6].

MACKE [7] replaces the one-electron orbitals by those which are automatically orthogonal to each other if the suitable variation conditions are fulfilled. We can describe the system as a whole with the ensemble of these functions which contain only one vector-variable constructed of the threespace coordinates. The energy expression in the case of a great number of particles gives the Thomas-Fermi energy. The procedure is intermediate between the Hartree-Fock and the statistical method. LADÁNYI [8] used it for concrete calculations, also.

Recently, GomBás [9] suggested a method by which he could notably simplify the SCF-procedure. Handling the electrons of a subshell uniformly we get as many equations as the number of the subshells. By this, the numerical work is greatly reduced and the accuracy is almost the same as originally.

In the following we suggest a method analogous to the one used in the statistical theory of fluids. By a suitable transformation of the energy operator we can obtain sufficiently correct results.

\section{Density transformation}

Let the normalized wave function of an arbitrary electron system be $\Psi\left(\mathfrak{r}_{1}, \mathfrak{r}_{2}, \ldots, \mathfrak{r}_{N}\right)$. The probability of finding any electron in the space element $d^{3} \mathrm{r}$ around the point defined by the vector $\mathrm{r}$ is

$$
\mathbf{P}(\mathfrak{r}) d^{3} \mathfrak{r}=\frac{d^{3} r}{N} \sum_{i=1}^{N} \int \Psi_{i} \Psi_{i}^{*} d v_{l},
$$

where

and

$$
\Psi_{i} \equiv \Psi\left(\mathrm{r}_{1}, \ldots, \mathrm{r}_{i}=\mathrm{r}, \ldots, \mathrm{r}_{N}\right)
$$

$$
d v_{i} \equiv d^{3} r_{1} \ldots d^{3} r_{i-1} d^{3} r_{i+1} \ldots d^{3} \mathfrak{r}_{N}
$$

$d v_{l} d^{3} r_{t}=d v$ is the whole configuration space element. Obviously, $N P(r)$ gives the charge density of the whole system.

Let us call density transformation the following:

$$
\varphi(\tau)=+\sqrt{\overline{\mathbf{P}(r)}}
$$

Henceforth we call $\varphi(\mathrm{r})$ density function. As $l \geqq P(r) \geqq 0 \varphi(r)$ is everywhere real and $1 \geq \varphi(x) \geqq 0$, it is easily seen that the density function is also normalized. Integrating its square with respect to $r$

$$
\frac{1}{N} \iint \sum_{i=1}^{N} \Psi_{i} \Psi_{i}^{*} d v_{i} d^{3} \mathfrak{r}=1
$$


putting in the $i$-th integrand $\mathfrak{r}_{i}$ and $d^{3} \mathfrak{r}_{i}$ in place of $\mathfrak{r}$ and $d^{3} \mathfrak{r}$, respectively, its value does not change but we get the norm of $\Psi$, the unity. Later, for the sake of simplicity we work with real eigenfunctions only. If $\Psi$ is a product of one-electron orbitals the density function is the following:

$$
\varphi(\mathfrak{r})=\sqrt{\frac{1}{N}-\sum_{i} \psi_{i}^{2}(\mathrm{r})}
$$

Let us examine how to transform an operator in order to get the correct expectation value with respect to the density function. Let $\mathbf{O}=\mathbf{O}\left(\mathfrak{r}_{1}, \mathfrak{r}_{2}, \ldots\right.$, $r_{N}$ ) be an arbitrary operator. The

$$
\omega(\mathfrak{r})=\frac{1}{N \varphi^{2}(\mathfrak{r})} \sum_{i} \int(\Psi \mathbf{O} \Psi)_{i} d v_{i}
$$

production operator satisfies the requirements and its expectation value with respect to the density function is just $\langle O\rangle$. As above, the index $i$ means that, ufter performing the operations, we put $\mathfrak{r}$ instead of $\mathfrak{r}_{i}$ in the parentheses. We oan prove analogously to our former concept that the choice in (3) is correct.

The use of $\omega(x)$ is inconvenient and we have to use the exact wave functions, also. Let us assume that $\boldsymbol{O}$ is the sum of the following one-electron operators:

$$
\begin{gathered}
\mathbf{O}=\sum_{i} \mathbf{O}^{i} \\
\mathbf{O}^{i}=\operatorname{grad}_{\mathfrak{r}_{i}} \text { or } \mathbf{O}^{i}=\mathbf{V}\left(\mathfrak{r}_{i}\right) .
\end{gathered}
$$

In this case $\Psi$ can be written in the form of a product: $\Psi=\Pi_{\psi_{i}}$ and the expectation value of the under operators

$$
N \operatorname{grad}_{\mathfrak{r}} \text { or } N \mathbf{V}(\mathrm{r})
$$

with respect to $\varphi(\mathrm{r})$ gives just the correct $\langle\mathbf{O}\rangle=\Sigma\left\langle\mathbf{O}^{i}\right\rangle$. Namely, the effect of the gradient operator according to the definition (1) of $\varphi$ is the following:

$$
\operatorname{grad}_{\mathfrak{r}} \varphi(\mathfrak{r})=\frac{1}{N \varphi(\mathfrak{r})} \sum_{i} \psi_{i}(\mathfrak{r}) \operatorname{grad}_{\mathfrak{r}} \psi_{i}(\mathfrak{r})
$$

and the expectation value is

$$
N \int \varphi(\mathfrak{r}) \operatorname{grad}_{\mathfrak{r}} \varphi(\mathfrak{r}) d^{3} \mathfrak{r}=\int \sum_{i} \psi_{i}(\mathfrak{r}) \operatorname{grad}_{\mathfrak{r}} \psi_{i}(\mathfrak{x}) d^{3} \mathfrak{r}
$$

Repeating the $\mathfrak{r}=\mathfrak{r}_{i}$ substitution we get $\left\langle\mathbf{O}^{i}\right\rangle .0$ n the other hand in (3) we wrote $\Sigma \mathbf{V}\left(\mathfrak{r}_{i}\right)$ in place of $O$ and so we got a special form of the $\omega(\mathfrak{r})$ operator 


$$
\omega(\mathfrak{r})=\frac{1}{N \varphi^{2}(\mathfrak{r})} \sum_{i} \psi_{i}(\mathrm{r}) \mathbf{V}(\mathrm{r}) \psi_{i}(\mathrm{r})=\mathbf{V}(\mathrm{r}) \cdot \frac{\sum \psi_{i}^{2}(\mathrm{r})}{N \varphi^{2}(\mathrm{r})}=\mathbf{V}(\mathrm{r})
$$

which means that the choice $\mathbf{O}^{i}=\mathbf{V}(\mathrm{r})$ is correct, too.

\section{Simplification of the Hartree method}

The Hamiltonian in atomic units can be written as follows:

$$
\mathbf{H}=-\frac{1}{2} \sum_{i=1}^{N} \operatorname{divgrad}_{\mathfrak{x}_{i}}-\sum_{i=1}^{N} \frac{Z}{r_{i}}+\sum_{i<j=2}^{N} \frac{1}{\left|\mathfrak{r}_{i}-{ }_{j}\right| \mathfrak{r}}
$$

Let us replace this by the following:

$$
\mathrm{H}=-\frac{N}{2} \operatorname{divgrad}_{\mathfrak{r}}-\frac{N Z}{r}+\left(\begin{array}{c}
N \\
2
\end{array}\right) \cdot \frac{1}{r} Y_{0}(r)^{*} .
$$

Here $1 / r \mathrm{Y}_{0}(r)$ means the solution of the spherically symmetrical Poissonequation:

$$
\frac{1}{r} Y_{0}(r)=\frac{1}{r} \int_{0}^{r} P^{2}\left(r^{\prime}\right) d r^{\prime}+\int_{r}^{\infty} \frac{1}{r^{\prime}} P^{2}\left(r^{\prime}\right) d r^{\prime}
$$

where $P^{2}$ is the spherically averaged electron density

$$
\begin{gathered}
P^{2}(r)=\frac{1}{N} \sum_{i} P_{i}^{2}(r)=\sum_{i=1}^{N} \frac{1}{N} \int_{0}^{2 \pi} \int_{0}^{\pi} \psi_{i}^{2}(r) r^{2} \sin \vartheta d \vartheta d \chi= \\
=\int_{0}^{2 \pi} \int_{0}^{\pi} \varphi^{2}(r) r^{2} \sin \vartheta d \vartheta d \chi
\end{gathered}
$$

We can immediately see that the second term in (7) corresponds to the (5) production operator and its expectation value is exactly the same as that of the attractive potential.

In the case of the kinetic energy operator we do not obtain exact values. Using the definition of the density function

$$
\operatorname{divgrad} \varphi(\mathfrak{r})=\frac{1}{N} \sum_{i}\left[\frac{\psi_{i}}{\varphi(\mathrm{r})} \operatorname{divgrad} \psi_{i}+\left(\operatorname{grad} \frac{\psi_{i}}{\varphi(\mathfrak{r})}, \operatorname{grad} \psi_{i}\right)\right]
$$

* The Pauli exclusion principle is not fulfilled with this operator. We can obtain the correct results only if we use those density functions which are constructed from antisymmetrical wave functions (these have to be known from other calculations). A priori calculations are possible, for example, with the aid of the pseudopotential included in the Hamiltonian (see Discussion). 
(For the sake of simplicity, here and later we do not designate the argument of the operator.) The scalar product occurring in the above expression can be brought to a simpler form by the use of the definition of $\varphi(r)$. So the expectation value of the approximate kinetic energy is the following:

$$
\begin{aligned}
E_{k}= & -\frac{1}{2} \int \varphi(\mathfrak{r}) N \operatorname{divgrad} \varphi(\mathfrak{r}) d^{3} \mathfrak{r}=-\frac{1}{2} \sum_{i} \int \psi_{i} \operatorname{divgrad} \psi_{i} d^{3} \mathfrak{r}- \\
& -\frac{1}{2} \sum_{i} \int\left[\left(\left|\operatorname{grad} \psi_{i}\right|\right)^{2}-\frac{\psi_{i}}{N \varphi^{2}(\mathrm{r})} \sum_{j} \psi_{j}\left(\operatorname{grad} \psi_{i}, \operatorname{grad} \psi_{j}\right)\right] d^{3} \mathfrak{r}
\end{aligned}
$$

Replacing $\mathfrak{r}=\mathfrak{r}_{i}$ the first term gives the expectation value of the exact kinetic energy. The second integrand may be brought to a simpler form, so we get

$$
\begin{aligned}
E_{k}-E_{k}(H F) & =\Delta E_{k}= \\
& =-\frac{1}{2 N} \sum_{i<j=2}^{N} \int \varphi^{-2}(\mathfrak{r})\left(\left|\psi_{j} \operatorname{grad} \psi_{i}-\psi_{i} \operatorname{grad} \psi_{j}\right|\right)^{2} d^{3} \mathfrak{r} .
\end{aligned}
$$

As the scalar product is always positive $A E_{k}<0$.

Let us now treat the Coulomb repulsive term of the Hamiltonian. It is well known that in the Hartree-Fock approximation we obtain the expectation value of the repulsive potential from the solution $1 / r_{i} Y_{0 j}$ of the spherically symmetrical Poisson-equation in the following way:

where

$$
E_{p r}(H F)=\sum_{i<j=2}^{N}(i j|g| i j),
$$

$$
(i j|g| i j)=\int_{0}^{\infty} P_{i}^{2}\left(r_{i}\right) \frac{1}{r_{i}} Y_{0 j} d r_{i}
$$

Here $P_{i}^{2}\left(r_{i}\right)$ means the radial probability density function of the $i$-th electron. The last term in the (7) operator gives the expectation value of the repulsive potential only approximately because on the basis of (8) and (9)

$$
\begin{gathered}
\left(\begin{array}{l}
N \\
2
\end{array}\right) \int_{0}^{\infty} P^{2}(r) \frac{1}{r} Y_{0}(r) d r=\frac{1}{N}\left(\begin{array}{l}
N \\
2
\end{array}\right) \int_{0}^{\infty} \sum_{i} P_{i}^{2}(r)\left[\frac{1}{r} \int_{0}^{r} \frac{1}{N} \sum_{j} P_{j}^{2}\left(r^{\prime}\right) d r^{\prime}+\right. \\
\left.\quad+\int_{r}^{\infty} \frac{1}{r^{\prime}} \cdot \frac{1}{N} \sum_{j} P_{j}^{2}\left(r^{\prime}\right) d r^{\prime}\right] d r=\frac{N-1}{2 N} \sum_{i, j=1}^{N}(i j|g| i j) .
\end{gathered}
$$

In the last step we made $r^{\prime}=r_{j}$ and the $r=r_{i}$ substitution in the internal and the external integrand, respectively. 
It is readily seen that the above sum gives the expectation value only approximately. The deviation is

$$
E_{p r}-E_{p r}(H F)=\Delta E_{p r}=\frac{1}{N}\left[\frac{N-1}{2} \sum_{i=1}^{N}(i i|g| i i)-\sum_{i<j=2}^{N}(i j|g| i j)\right],
$$

which can be easily proved by the aid of simple transformations. We cannot tell anything connected with the sign of $\Delta E_{p r}$; later we shall see two examples where it is positive.

In order to make an estimation of the errors let us calculate the value of (10) and (11) in the case of the Ne-atom. Using $\mathrm{H}$-type orbitals, the charge distribution and $\varphi$ also is spherically symmetrical. The kinetic energy using the density function obtained from the above orbitals is $E_{k}=13 \alpha^{2} / 4$, its error relative to the one-electron calculation is $\Delta E_{k}=-0.084 \alpha^{2}(-2.6 \%)$, where $\alpha$ means the variation parameter of the wave functions. The repulsive potential is $E_{p r}=8.42 \alpha$, its error is $\Delta E_{p r}=0.35 \alpha(4.15 \%)$. The attractive potential is $-13 \alpha Z / 2$, its error is zero so the minimum of the total energy is at $\alpha==8.7$, its value is $E=-283.9$, the error is $\Delta E=-3.4(1.43 \%)$. If we choose $e^{-5^{\alpha r}}, e^{-4^{\alpha r}}, \ldots, e^{-\alpha r}$ as orbitals the kinetic energy is $55 \alpha^{2}$ the error, calculating (10) graphically, is about $-0.138 \alpha^{2}(0.25 \%)$. The repulsive potential is $84.5 \alpha$, its error is $0.465 \alpha(0.55 \%)$, the attractive potential is $-30 \alpha Z$ so the minimum energy $(\alpha=1.96)$ is -197.0 , its error is $0.38(0.19 \%)$. The total energy of the $\mathrm{Ne}$ obtained experimentally is - 129.5 (all values in atomic units). Because of the omission of the Pauli exclusion principle the absolute values of the above data are incorrect but we make a comparison between the energy calculated by the one and the many-dimensional function, respectively. It is interesting that the errors are different in the case of the two types of orbitals.

\section{Discussion}

The method outlined above is applicable in two ways. First, we can construct three-dimensional wave function with the aid of the many-dimensional eigenfunctions obtained otherwise. This makes a great simplification of further calculations possible. The one-electron orbitals of Hartree-type are not suitable for this purpose. The function constructed by the density transformation will be spherically symmetrical in the case of a closed shell but difficulties will arise in the choice of the kinetic energy operator. Namely, the Laplace-operator is the sum of a radial and a spherical term:

$$
\begin{gathered}
\Delta=\Delta_{r}+\Delta_{i x} \\
\Delta_{r}=\frac{\partial^{2}}{\partial r^{2}}+\frac{2}{r} \cdot \frac{\partial}{\partial r}, \quad \Delta_{\vartheta x}=\frac{1}{r^{2}}\left(\frac{\partial^{2}}{\partial \vartheta^{2}}+\operatorname{ctg} \vartheta \cdot \frac{\partial}{\partial \vartheta}+\frac{1}{\sin ^{2} \vartheta} \cdot \frac{\partial^{2}}{\vartheta \chi^{2}}\right) .
\end{gathered}
$$


If the operator $-N \Delta_{r} / 2$ acts on the density function (the effect of the second term is zero) we neglect the angle-dependent term occurring in the HartreeFock approximation, also:

$$
\sum_{i} \iiint \psi_{i} \Delta_{\vartheta \chi} \psi_{i} r^{2} \sin \vartheta d r d \vartheta d \chi=\sum_{i} \int l(l+1) \psi_{i}^{2} d r
$$

The neglect of this term gives a considerable error. $\psi_{i}$ appears in the above expression weighted with $l(l+1)$ and constructing the density function we consider it always with the same weight. So we should have an operator which produces a weighted sum from the non-weighted $\varphi$. If we do not use the original wave function this is not possible. The solution should have been a production operator corresponding to (3) but this is so complicated that the method would lose its value. The Ritz variation procedure is a possible way to obtain concrete results with the aid of the density function. We approach the $\varphi$ by a suitable expression and we calculate the minimum of the energy so we can obtain the best parameters of our trial function. The Pauli exclusion principle is not included in the density function; this is to be considered in some suitable subsidiary condition. Most convenient is the pseudopotential used in the statistical theory of the atom [10]. This is an energy-like production operator which, added to the Hamiltonian, does not let all the electrons drop into the lowest energy states.

The method is primarily convenient in the treatment of the ground state. In calculating the atomic spectra several difficulties arise. A given configuration is to be written in the best way with the aid of the one-electron orbitals. As the density transformation does not make any difference between the individual properties of the electrons, the excited states belonging to different symmetries are indistinguishable. According to similar reasons the formulation of the exchange of electrons is also problematic. As the density function is always real in the case of non-stationary problems, it is useless.

One can expect that the most correct results are obtained in the spherically symmetrical case. This is suggested by the vast reduction of the error with the use of spherically symmetrical orbitals. So the value of the method is greatest for the calculation of this case.

\section{REFERENCES}

1. P. Gomвás, Die statistische Theorie des Atoms und ihre Anwendungen, Springer, Wien, 1949.

2. I. Prigogine, The molecular theory of solutions, North-Holland Publ. Comp., Amsterdam, 1957.

3. R. MCWeENY, Proc. Roy. Soc., A223 63 and 306, 1954.

4. P.-O. Löwdin, Phys. Rev., 97, 1474, 1955.

5. L. C. R. Alfred, Phys. Rev., 121, 1275, 1961. 
6. R. S. Coleman, Canad. Math. Bull., 4, 209, 1961.

7. W. Macke, Phys. Rev., 100, 992, 1955.

8. K. Ladányi, Acta Phys. Hung., 9, 115, 1958.

9. P. Gombás, Theor. Chim. Acta, 5, 112, 1966.

I0. P. Gombás, Z. Phys., 118, 164, 1941.

11. P. Gombás, Acta Phys. Hung., 1, 258, 1952.

\author{
ПРИБЛИЖЕНИЕ ПРОБЛЕМЫ МНОГИХ ЭЛЕКТРОНОВ \\ ПУТЕМ ЕДИНОЙ ДИСКУССИИ ЗАРЯДНОГО ОБЛАКА \\ r. HAPAK-CABO \\ Рез юм е
}

Подобно образованию редуцированных матриц плотности первого ранга из решекия проблемы многих тел с $3 \mathrm{~N}$ - переменными можно производить так называемые функции плотности с тремя переменными. При помощи этих функций энергия атома вычисляется с приближенной точностью при условии выбора оператора Гамильтона в соответствующей форме. Отличие от метода Хартри-- Фока в зависимости от вида волновой функции несколько десятых доли процента или несколько процентов. Приближение наиболее точно и просто в случае.сферической симметрии. Функция плотности применима только для дискуссии стационарных проблем. 